\title{
A simple deep breathing test reveals altered cerebral autoregulation in type 2 diabetic patients
}

\author{
C. M. Brown • H. Marthol • U. Zikeli • \\ D. Ziegler • M. J. Hilz
}

Received: 13 November 2007 / Accepted: 21 January 2008 / Published online: 29 February 2008

(C) Springer-Verlag 2008

\begin{abstract}
Aims/hypothesis Patients with diabetes mellitus have an increased risk of stroke and other cerebrovascular complications. The purpose of this study was to evaluate the autoregulation of cerebral blood flow in diabetic patients using a simple method that could easily be applied to the clinical routine screening of diabetic patients.

Methods We studied ten patients with type 2 diabetes mellitus and 11 healthy volunteer control participants. Continuous and non-invasive measurements of blood pressure and cerebral blood flow velocity were performed during deep breathing at $0.1 \mathrm{~Hz}$ (six breaths per minute). Cerebral autoregulation was assessed from the phase shift angle between breathing-induced $0.1 \mathrm{~Hz}$ oscillations in mean blood pressure and cerebral blood flow velocity.
\end{abstract}

C. M. Brown · H. Marthol • U. Zikeli • M. J. Hilz

Department of Neurology, University of Erlangen-Nuremberg,

Erlangen, Germany

C. M. Brown ( $\square)$

Department of Medicine, Division of Physiology,

University of Fribourg,

Rue du Musée 5,

1700 Fribourg, Switzerland

e-mail: clivemartin.brown@unifr.ch

M. J. Hilz

Departments of Neurology, Medicine and Psychiatry,

New York University School of Medicine,

New York, NY, USA

D. Ziegler

Institute for Clinical Diabetes Research, German Diabetes Center,

Leibniz Center at the Heinrich Heine University,

Düsseldorf, Germany
Results The controls and patients all showed positive phase shift angles between breathing-induced $0.1 \mathrm{~Hz}$ blood pressure and cerebral blood flow velocity oscillations. However, the phase shift angle was significantly reduced $(p<0.05)$ in the patients $\left(48 \pm 9^{\circ}\right)$ compared with the controls $\left(80 \pm 12^{\circ}\right)$. The gain between $0.1 \mathrm{~Hz}$ oscillations in blood pressure and cerebral blood flow velocity did not differ significantly between the patients and controls.

Conclusions/interpretation The reduced phase shift angle between oscillations in mean blood pressure and cerebral blood flow velocity during deep breathing suggests altered cerebral autoregulation in patients with diabetes and might contribute to an increased risk of cerebrovascular disorders.

Keywords Autonomic nervous system · Blood pressure regulation $\cdot$ Cerebral autoregulation $\cdot$ Diabetes .

Heart rate variability

\section{Abbreviation}

CBFV cerebral blood flow velocity

\section{Introduction}

Patients with diabetes mellitus have an increased risk of cerebrovascular complications, including stroke [1-3]. The association between diabetes and stroke is probably related, at least in part, to impaired nitric oxide synthase-dependent responses of cerebral blood vessels $[4,5]$. An additional risk factor contributing to the incidence of strokes in diabetic patients is cardiovascular autonomic neuropathy, which is a common complication of diabetes mellitus and is itself associated with increased morbidity and mortality [6, 7].

Cerebral autoregulation is the phenomenon by which cerebral blood flow remains near-constant despite changes 
in systemic blood pressure, provided that mean blood pressure remains within certain limits, usually $50-150 \mathrm{mmHg}$ [8]. Vascular dysfunction and autonomic neuropathy are likely to contribute to compromised cerebral autoregulation in patients with type 2 diabetes, thereby enhancing the risk of cerebrovascular complications [9]. Testing of cerebral autoregulation is often performed by measuring responses of the cerebral blood vessels to blood pressure changes induced either by pharmacological (e.g. phenylephrine infusion) or mechanical (e.g. release of a thigh cuff) means [10]. In a previous study we assessed cerebral autoregulation in diabetic patients by mechanically stimulating the carotid baroreceptors using oscillatory neck suction [11]. We demonstrated that, during neck suction, the patients showed a decreased phase relationship between fluctuations in blood pressure and cerebral blood flow velocity (CBFV), suggesting altered cerebral autoregulation [11]. While techniques such as neck suction are useful research tools for studying cardiovascular and cerebrovascular regulation, their clinical application is somewhat limited, largely as a result of the specialised equipment involved, as well as the complex analysis techniques required. An alternative and simple method of measuring cerebral autoregulation was introduced by Diehl et al. [12]. Their technique involves generating sinusoidal oscillations in blood pressure by slow breathing at six breaths per minute $(0.1 \mathrm{~Hz})$. In healthy individuals these oscillations in blood pressure are transmitted to the cerebral blood flow but shifted to the left with a phase angle of about $70^{\circ}$ [12]. In patients with known autoregulatory disturbances this phase shift angle is reduced, suggesting a more passive transfer of blood pressure fluctuations onto the cerebral vasculature [12]. This technique of assessing cerebral autoregulation requires no specialised equipment for inducing blood pressure changes and relies only on the patient's ability to breathe slowly and deeply. As such, it is a potentially useful method for clinical application in diabetic patients who might have subtle cerebrovascular disturbances.

The aim of this study was to evaluate the slow breathing method as a clinical test of autoregulation in type 2 diabetic patients. We hypothesised that diabetic patients would show a reduction in the phase shift between $0.1 \mathrm{~Hz}$ breathinginduced oscillations in blood pressure and $\mathrm{CBFV}$, indicating impaired cerebral autoregulation.

\section{Methods}

We studied ten patients (two women, eight men), aged $58 \pm 2$ years, who had a confirmed diagnosis of type 2 diabetes mellitus according to the criteria of the American Diabetes Association and the World Health Organization. Their average height was $173 \pm 3 \mathrm{~cm}$, average weight $87 \pm 6 \mathrm{~kg}$ and BMI $28.8 \pm 1.7 \mathrm{~kg} / \mathrm{m}^{2}$. Their mean duration of diabetes was $79 \pm 16$ months. Five patients were being treated with insulin and seven patients were on oral glucose-lowering drugs such as sulfonylureas or metformin. At the time of testing, the $\mathrm{HbA}_{1 \mathrm{c}}$ levels of the patients ranged from $6.3 \%$ to $7.7 \%$, while blood glucose ranged from 5.1 to $6.7 \mathrm{mmol} / \mathrm{l}$. All patients underwent a battery of standard cardiovascular reflex tests, including assessment of heart rate variability at rest and during metronomic breathing, the Valsalva manoeuvre and active standing [13]. Based on these tests, six of the patients fulfilled the criteria of cardiovascular autonomic neuropathy (defined as two or more abnormal test results). None of the patients, however, showed any overt symptoms or signs of diabetic neuropathy. For $24 \mathrm{~h}$ prior to testing, all patients self-monitored their blood glucose at least three times $1 \mathrm{~h}$ after meals. The postprandial blood glucose levels were all below $7.8 \mathrm{mmol} / \mathrm{l}$.

We also tested 11 healthy controls (four women and seven men). Their average age was $54 \pm 3$ years, average height $168 \pm 2 \mathrm{~cm}$, average weight $76 \pm 5 \mathrm{~kg}$ and average BMI $26.6 \pm 1.3 \mathrm{~kg} / \mathrm{m}^{2}$. The controls were recruited among healthy friends or relatives of staff members and patients.

None of the participants (patients and controls) had a history of chronic alcohol abuse, carcinoma, myopathy, hyper- or hypothyroidism, arterial hypertension, cardiac arrhythmia, diagnosed atherosclerosis, previous organ transplantation, renal disease or any other condition that would interfere with their ability to participate in the study. Apart from insulin or glucose-lowering drugs in the diabetic patients, none of the participants was on any other medication known to affect cardiovascular or autonomic regulation. All participants were asked not to consume nicotine, caffeine or alcohol for $24 \mathrm{~h}$ before the experiments and were studied at least $4 \mathrm{~h}$ after consuming a light meal.

The study was carried out in accordance with the Declaration of Helsinki and its design was approved by the University of Erlangen-Nuremberg Ethics Committee. Written informed consent was obtained from each study participant.

Procedure The tests were performed in a quiet, temperaturecontrolled $\left(24^{\circ} \mathrm{C}\right)$ room. The patients and controls were studied in a supine position. Instrumentation for beat-to-beat cardiovascular and cerebrovascular monitoring was attached and the measurements were started after about 45 min once the signals had stabilised. The patients and controls were then instructed to breathe deeply for $3 \mathrm{~min}$ at the rate of six breaths per minute ( $5 \mathrm{~s}$ inspiration and $5 \mathrm{~s}$ expiration) by following combined visual and auditory stimuli. This standard challenge manoeuvre induces sinusoidal oscillations in arterial blood pressure and CBFV at a frequency of $0.1 \mathrm{~Hz}$ (six per minute) [12].

We continuously monitored the electrocardiographic RR intervals and non-invasive beat-to-beat blood pressure at 
Table 1 Baseline cardiovascular parameters in controls and patients with type 2 diabetes

\begin{tabular}{llll}
\hline Parameter & $\begin{array}{l}\text { Controls } \\
(n=11)\end{array}$ & $\begin{array}{l}\text { Diabetic patients } \\
(n=10)\end{array}$ & $p$ value \\
\hline $\begin{array}{l}\text { Heart rate (beats per min) } \\
\begin{array}{l}\text { Systolic blood pressure } \\
(\mathrm{mmHg})\end{array}\end{array}$ & $63 \pm 3$ & $73 \pm 4$ & 0.10 \\
$\begin{array}{l}\text { Diastolic blood pressure } \\
(\mathrm{mmHg})\end{array}$ & $74 \pm 3$ & $72 \pm 2$ & 0.73 \\
\hline
\end{tabular}

the left radial artery using applanation tonometry calibrated by a brachial blood pressure cuff (Colin Pilot; Colin Medical, San Antonio, TX, USA). Respiratory frequency was monitored by calibrated electrical inductance plethysmography (Respitrace Calibrator; Ambulatory Monitoring, Ardsley, NY, USA) with two respiratory belts placed around the chest and abdomen.

We recorded CBFV using transcranial Doppler ultrasonography (Multidop XL; DWL, Sipplingen, Germany) at the proximal segment of the middle cerebral artery, insonated through the temporal window approximately $1 \mathrm{~cm}$ above the zygomatic arch at a depth of 40-60 mm using $2 \mathrm{MHz}$ probes. After optimising the Doppler signal by standard methods, the probe was stabilised in a fixed position using a headband. Expiratory air was sampled via a nasal cannula and end-tidal $\mathrm{CO}_{2}\left(\mathrm{PETCO}_{2}\right)$ measured by infrared absorption (Colin Pilot; Colin Medical).

Data analysis All data were digitised using a custommade analogue-to-digital converter at a sampling rate of $300 \mathrm{~Hz}$, fed to a microcomputer (Macintosh Powerbook; Apple, Cupertino, CA, USA), and stored for off-line analysis. The QRS complexes of the electrocardiogram were identified using a C-language program. Beat-to-beat mean values of RR intervals, mean arterial pressure and CBFV were calculated automatically and interpolated linearly between adjacent values to construct a corresponding continuous time series. From the time series, data segments of $90 \mathrm{~s}$ with the most stable $\mathrm{PETCO}_{2}$ values were analysed.

Oscillations in mean arterial pressure, RR intervals and CBFV were evaluated by autoregressive power spectrum analysis. Dynamic cerebral autoregulation was determined by using cross-spectral analysis to characterise the relationship between breathing-induced oscillations in blood pressure and CBFV. We calculated the gain and the phase relationship between mean blood pressure and CBFV oscillations at $0.1 \mathrm{~Hz}$ to quantify the transmission of $0.1 \mathrm{~Hz}$ breathing-induced blood pressure oscillations onto the cerebral vasculature.
Statistical analysis All values are reported as mean \pm SEM. Mean values of all the recorded signals, frequency-domain responses and parameters of cerebral autoregulation were compared between controls and patients using a two-sided $t$ test with Welch correction for independent samples. Because of their skewed distributions, powers of RR intervals, blood pressure and $\mathrm{CBFV}$ were analysed after natural logarithmic transformation. Statistical significance was accepted at $p<0.05$. Statistical tests were performed using commercially available software (Instat version 3.01; Graphpad Software, San Diego, CA, USA).

\section{Results}

Resting values of the cardiovascular variables recorded during the baseline period are listed in Table 1. The basal cardiovascular state was similar for patients and controls, although the resting heart rate tended to be slightly higher in the patients than in the control participants.

Each of the diabetic patients and healthy controls was able to perform the slow breathing test at six breaths per minute. Slow breathing at $0.1 \mathrm{~Hz}$ induced oscillations at $0.1 \mathrm{~Hz}$ in RR interval, blood pressure and CBFV in all controls and patients. End-tidal $\mathrm{CO}_{2}$ levels during deep breathing were $32.1 \pm 1.7 \mathrm{mmHg}$ in the controls and $32.2 \pm 2.9 \mathrm{mmHg}$ in the patients $(p=0.98)$. Figure 1 shows the power of breathing-induced breathing oscillations in RR interval at $0.1 \mathrm{~Hz}$. In the diabetic patients, RR interval variability at $0.1 \mathrm{~Hz}$ during $0.1 \mathrm{~Hz}$ breathing was significantly less than in the control participants $(p<0.01)$. The powers of mean blood pressure and CBFV variabilities at $0.1 \mathrm{~Hz}$ during slow breathing are shown in Fig. 2. Although the power of breathing-induced $0.1 \mathrm{~Hz}$ mean blood pressure oscillations was slightly greater in the controls than in the patients, the difference was not significant $(p=0.39)$.

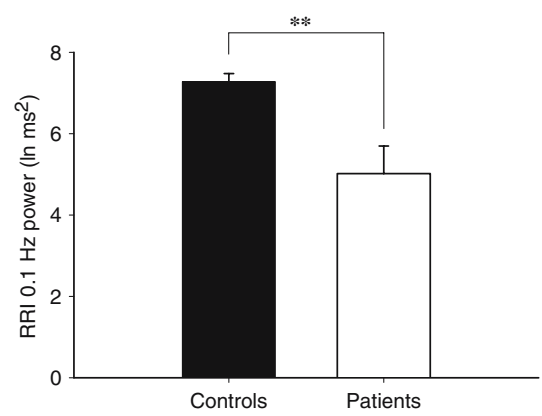

Fig. 1 Powers of low-frequency variability in RR interval (RRI) during slow breathing at $0.1 \mathrm{~Hz}$ in the 11 healthy controls and ten diabetic patients. Values are means \pm SEM. The variability of RRI was significantly less in the patients than in the controls $(* * p<0.01)$ 
a

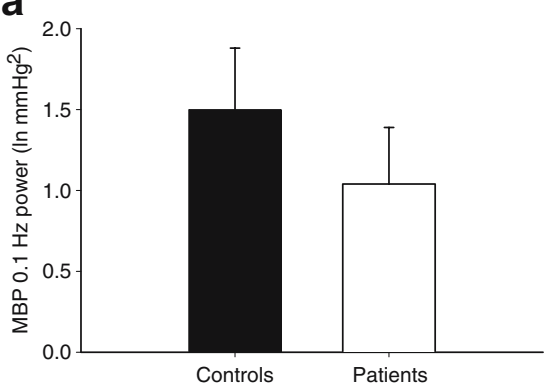

b

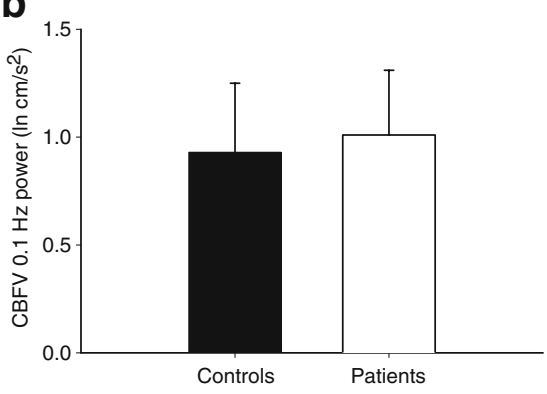

Fig. 2 Powers of low-frequency $(0.1 \mathrm{~Hz})$ variability (mean \pm SEM) in a mean blood pressure (MBP) and $\mathbf{b}$ CBFV during slow breathing at $0.1 \mathrm{~Hz}$ in the 11 healthy controls and ten diabetic patients. There were no significant differences between controls and patients in the variability of either mean blood pressure or cerebral blood flow velocity

There was also no difference in the power of slow breathinginduced $0.1 \mathrm{~Hz} \mathrm{CBFV}$ oscillations between the controls and patients $(p=0.87)$. Cross-spectral analysis revealed that the gain between $0.1 \mathrm{~Hz}$ oscillations between blood pressure and CBFV was not significantly different $(p=0.13)$ between the controls $\left(0.79 \pm 0.07 \mathrm{~cm} \mathrm{~s}^{-1} \mathrm{mmHg}^{-1}\right)$ and patients $\left(1.10 \pm 0.18 \mathrm{~cm} \mathrm{~s}^{-1} \mathrm{mmHg}^{-1}\right)$. In all controls and patients, cerebral blood flow oscillations showed a positive phase shift in relation to the blood pressure oscillations. The phase shift between $0.1 \mathrm{~Hz}$ blood pressure and CBFV oscillations was, however, significantly lower in the patients than in the controls (Fig. 3). There was no correlation between the phase shift and RR interval variability during deep breathing.

\section{Discussion}

Although several techniques are available for assessment of cerebral autoregulation, some are unsuitable for routine clinical use because they are too cumbersome, require special equipment or involve discomfort to the patient. In the present study we evaluated the slow breathing technique, introduced by Diehl et al. [12], as a clinical test of cerebral autoregulation in patients with type 2 diabetes. We found that all patients and controls were able to maintain the slow breathing frequency of six breaths per minute, which induced $0.1 \mathrm{~Hz}$ fluctuations in blood pressure that were transferred onto the
$\mathrm{CBFV}$. In the healthy controls, the CBFV oscillations showed a positive phase shift, i.e. to the left of blood pressure oscillations, by about $80^{\circ}$. In the type 2 diabetic patients the phase shift of the oscillations in CBFV was substantially lower, indicating altered cerebral autoregulation.

Blood pressure oscillations induced by breathing are predominantly due to the mechanical effects of fluctuations in cardiac stroke volume [14]. Slow breathing at six cycles per minute $(0.1 \mathrm{~Hz})$ generates oscillations in blood pressure at the same frequency that are transmitted onto the CBFV [12]. Because the mechanisms of cerebral autoregulation resemble a high-pass filter, the resulting CBFV oscillations are shifted to the left [12]. This phase shift can be explained by the time lag with which the cerebrovascular resistance vessels respond to changes in cerebral perfusion pressure [15]. Diehl et al. [12] demonstrated that healthy individuals had a phase shift of approximately $70^{\circ}$, with a lower normal limit of $30^{\circ}$. In contrast, in patients with disturbed cerebral autoregulation the phase shift was considerably reduced [12].

In our diabetic patients the phase shift between $0.1 \mathrm{~Hz}$ breathing-induced oscillations in blood pressure and CBFV was less than in the healthy controls, suggesting a slower adaptation of the cerebral resistance vessels and impairment of autoregulation. On an individual basis, three of our diabetic patients but none of our healthy controls had phase shift values that were less than the lower normal limit $\left(30^{\circ}\right)$ that was previously defined by Diehl et al. [12]. Furthermore, four of our patients $(40 \%)$ had values that were less than the lowest value that we measured in our healthy controls. These findings are in line with our previous observation that the phase shift between blood pressure and CBFV was reduced in type 2 diabetic patients during carotid baroreceptor stimulation using neck suction [11]. One limitation of that study was that, due to baroreflex dysfunction, carotid baroreceptor stimulation did not

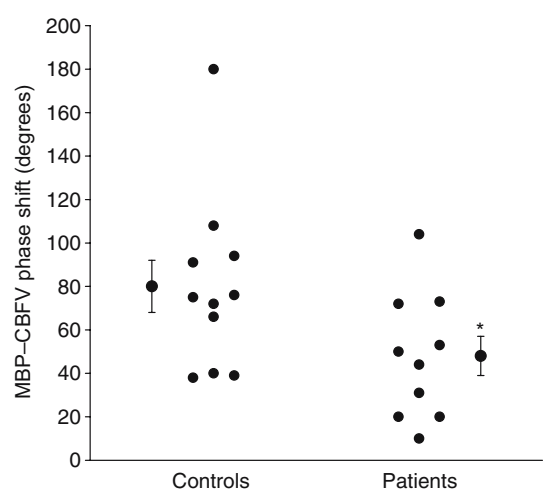

Fig. 3 Individual and mean \pm SEM phase shift angles between breathing-induced $0.1 \mathrm{~Hz}$ mean blood pressure (MBP) and CBFV oscillations in the 11 healthy controls and ten diabetic patients. The phase shift was significantly reduced in patients $\left({ }^{*} p<0.05\right)$ compared with controls, indicating altered cerebral autoregulation 
stimulate fluctuations in blood pressure in the diabetic patients. The lack of blood pressure change (the input to cerebral autoregulation) during neck suction complicates interpretation of the results. Our current approach avoids this problem because the blood pressure oscillations generated by breathing are largely mechanical, rather than reflex, in origin. It is therefore possible to induce blood pressure oscillations in diabetic patients, even if they have cardiovascular autonomic dysfunction. Further advantages of the slow breathing method for assessing autoregulation are that it is simple, does not require highly specialised equipment and depends only on the patients' ability to regulate their breathing for only a few minutes. The technique is therefore ideally suited as a clinical test of cerebral autoregulation in diabetic patients.

The gain between $0.1 \mathrm{~Hz}$ oscillations in blood pressure and CBFV was similar in our diabetic patients and controls. Overall dampening of $0.1 \mathrm{~Hz}$ breathing-induced blood pressure fluctuations onto the cerebral vasculature did not, therefore, appear to be impaired in the diabetic patients. Thus, the autoregulatory disturbances in our patients were relatively subtle. Previous studies have also reported relatively mild alterations in cerebral blood flow autoregulation in patients with diabetes $[16,17]$. In some diabetic patients, impaired autoregulation might contribute to orthostatic hypotension [9], which is a known risk factor for ischaemic stroke [18]. Studies suggest that assessment of cerebral autoregulation can identify patients with increased risk of stroke [19, 20]. Diagnosis of autoregulatory disturbances in patients with diabetes is therefore particularly important, even in the early stages of the disease. While our deep breathing method is able to identify altered cerebral autoregulation in diabetic patients, there is a clear need for larger-scale studies that compare our technique with neuroimaging techniques such as magnetic resonance imaging, perfusion positron emission tomography and transcranial B-mode ultrasound examinations, to determine whether altered phase shifts precede, parallel or correlate with neuroimaging signs of diabetic cerebrovascular complications.

Several factors probably contribute to impaired autoregulation in type 2 diabetic patients. The presence of vascular abnormalities in diabetes is well established and is characterised by increased vascular permeability [21], endothelial dysfunction [22] and capillary basement membrane thickening [23]. Many diabetic patients also have autonomic dysfunction, affecting sympathetic and parasympathetic regulation of the cardiovascular system [24]. Several of our patients had cardiovascular autonomic neuropathy as determined using standard tests of autonomic function. Furthermore, heart rate variation during $0.1 \mathrm{~Hz}$ breathing was markedly reduced in our diabetic patients compared with the controls, suggesting impaired parasympathetic regulation. The role of the autonomic nervous system in cerebral autoregulation is, however, controversial $[25,26]$. Studies in animals suggest that parasympathetic activation results in cerebrovascular vasodilatation [27]. In the present study there was no correlation between heart rate variability during deep breathing (an index of parasympathetic function) and the phase relation between mean blood pressure and CBFV. Nevertheless, we cannot exclude autonomic dysfunction as a possible contributor to impaired autoregulation in our diabetic patients.

An important methodological point concerns the use of transcranial Doppler for measurements of cerebral blood flow. Changes in CBFV reflect changes in cerebral blood flow only if the diameter of the insonated artery remains constant. There is considerable experimental data suggesting that the diameter of the mid-cerebral artery remains stable during rapid alterations in arterial pressure [28] and during orthostatic stress [29]. We are therefore confident that the changes in $\mathrm{CBFV}$ that we measured reflect actual changes in cerebral blood velocity and were not influenced by changes in the diameter of the insonated artery.

Our findings suggest that patients with type 2 diabetes have subtle disturbances in cerebral autoregulation that can be identified by measuring fluctuations in blood pressure and CBFV during a deep breathing test. Long-term clinical studies are required to determine the progression of autoregulatory disturbances and their potential link to the heightened risk of stroke in diabetic patients.

Duality of interest The authors declare that there is no duality of interest associated with this manuscript.

\section{References}

1. Kothari V, Stevens RJ, Adler AI et al (2002) UKPDS 60: risk of stroke in type 2 diabetes estimated by the UK Prospective Diabetes Study risk engine. Stroke 33:1776-1781

2. Lavy S, Melamed E, Cahane E, Carmon A (1973) Hypertension and diabetes as risk factors in stroke patients. Stroke 4:751-759

3. Stegmayr B, Asplund K (1995) Diabetes as a risk factor for stroke. A population perspective. Diabetologia 38:1061-1068

4. Fujii K, Heistad DD, Faraci FM (1992) Effect of diabetes mellitus on flow-mediated and endothelium-dependent dilatation of the rat basilar artery. Stroke 23:1494-1498

5. Mayhan WG (1989) Impairment of endothelium-dependent dilatation of cerebral arterioles during diabetes mellitus. Am J Physiol 256:H621-H625

6. Vinik AI, Ziegler D (2007) Diabetic cardiovascular autonomic neuropathy. Circulation 115:387-397

7. Toyry JP, Niskanen LK, Lansimies EA, Partanen KP, Uusitupa MI (1996) Autonomic neuropathy predicts the development of stroke in patients with non-insulin-dependent diabetes mellitus. Stroke 27:1316-1318

8. Aaslid R, Lindegaard KF, Sorteberg W, Nornes H (1989) Cerebral autoregulation dynamics in humans. Stroke 20:45-52

9. Mankovsky BN, Piolot R, Mankovsky OL, Ziegler D (2003) Impairment of cerebral autoregulation in diabetic patients with 
cardiovascular autonomic neuropathy and orthostatic hypotension. Diabet Med 20:119-126

10. Tiecks FP, Lam AM, Aaslid R, Newell DW (1995) Comparison of static and dynamic cerebral autoregulation measurements. Stroke 26:1014-1019

11. Marthol H, Brown CM, Zikeli U et al (2006) Altered cerebral regulation in type 2 diabetic patients with cardiac autonomic neuropathy. Diabetologia 49:2481-2487

12. Diehl RR, Linden D, Lucke D, Berlit P (1995) Phase relationship between cerebral blood flow velocity and blood pressure. A clinical test of autoregulation. Stroke 26:1801-1804

13. Ewing DJ, Clarke BF (1982) Diagnosis and management of diabetic autonomic neuropathy. BMJ (Clin Res Ed) 285:916-918

14. Zhang R, Iwasaki K, Zuckerman JH, Behbehani K, Crandall CG, Levine BD (2002) Mechanism of blood pressure and R-R variability: insights from ganglion blockade in humans. J Physiol 543:337-348

15. Hughson RL, Edwards MR, O’Leary DD, Shoemaker JK (2001) Critical analysis of cerebrovascular autoregulation during repeated head-up tilt. Stroke 32:2403-2408

16. Asil T, Utku U, Balci K, Uzunca I (2007) Changing cerebral blood flow velocity by transcranial Doppler during head up tilt in patients with diabetes mellitus. Clin Neurol Neurosurg 109:1-6

17. Bentsen N, Larsen B, Lassen NA (1975) Chronically impaired autoregulation of cerebral blood flow in long-term diabetics. Stroke 6:497-502

18. Eigenbrodt ML, Rose KM, Couper DJ, Arnett DK, Smith R, Jones D (2000) Orthostatic hypotension as a risk factor for stroke: the atherosclerosis risk in communities (ARIC) study, 1987-1996. Stroke 31:2307-2313

19. Gur AY, Bova I, Bornstein NM (1996) Is impaired cerebral vasomotor reactivity a predictive factor of stroke in asymptomatic patients. Stroke 27:2188-2190
20. Silvestrini M, Vernieri F, Pasqualetti $P$ et al (2000) Impaired cerebral vasoreactivity and risk of stroke in patients with asymptomatic carotid artery stenosis. JAMA 283:2122-2127

21. Joyner WL, Mayhan WG, Johnson RL, Phares CK (1981) Microvascular alterations develop in Syrian hamsters after the induction of diabetes mellitus by streptozotocin. Diabetes 30:93-100

22. Hogikyan RV, Galecki AT, Pitt B, Halter JB, Greene DA, Supiano MA (1998) Specific impairment of endothelium-dependent vasodilation in subjects with type 2 diabetes independent of obesity. J Clin Endocrinol Metab 83:1946-1952

23. Johnson PC, Brendel K, Meezan E (1982) Thickened cerebral cortical capillary basement membranes in diabetics. Arch Pathol Lab Med 106:214-217

24. Sanya EO, Brown CM, Dutsch M, Zikeli U, Neundorfer B, Hilz MJ (2003) Impaired cardiovagal and vasomotor responses to baroreceptor stimulation in type II diabetes mellitus. Eur J Clin Invest 33:582-588

25. Heistad DD, Marcus ML (1978) Evidence that neural mechanisms do not have important effects on cerebral blood flow. Circ Res 42:295-302

26. Zhang R, Zuckerman JH, Iwasaki K, Wilson TE, Crandall CG, Levine BD (2002) Autonomic neural control of dynamic cerebral autoregulation in humans. Circulation 106:1814-1820

27. Talman WT, Corr J, Nitschke DD, Wang D (2007) Parasympathetic stimulation elicits cerebral vasodilatation in rat. Auton Neurosci 133:153-157

28. Newell DW, Aaslid R, Lam A, Mayberg TS, Winn HR (1994) Comparison of flow and velocity during dynamic autoregulation testing in humans. Stroke 25:793-797

29. Serrador JM, Picot PA, Rutt BK, Shoemaker JK, Bondar RL (2000) MRI measures of middle cerebral artery diameter in conscious humans during simulated orthostasis. Stroke 31:16721678 